\title{
Research on evaluation method of bearing capacity of existing bridge based on static load test
}

\author{
Qingcheng Yang ${ }^{1}$, Yufeng Ning ${ }^{2}$ and Weiwei Sun ${ }^{1 *}$, Shunchao Cheng ${ }^{2 *}$ \\ ${ }^{1}$ College of Civil Engineering, Southwest Forestry University, Kunming, Yunnan, 650224, China \\ ${ }^{2}$ College of Civil Engineering, Southwest Forestry University, Kunming, Yunnan, 650224, China
}

\begin{abstract}
The author carried out static load test research on a prestressed concrete rigid frame bridge. Under the action of static load, the most unfavorable section JM3 on the beam body was measured. The stress and deflection values at each measuring point were measured. The results show that the check coefficient of stress and deflection at each measuring point of the box girder does not exceed the standard limit value 1, the relative residual strain or deformation at each measuring point does not exceed the standard limit value $20 \%$, and the overall bearing capacity of the bridge structure meets the normal use requirements of the design load (highway -I class).
\end{abstract}

\section{Introduction}

Static load test of bridge beam is mainly to measure the stress and structural deformation of each control section of bridge structure under static load, so as to determine whether the actual working state of bridge conforms to the design expectation. It is the most direct and effective way to test the performance of bridge and the strength and stiffness of bridge structure.

\section{Project profile}

In this paper, a national highway bridge in Yunnan province is used as the engineering support. The bridge is located in the grand Canyon area with steep terrain and adopts a three-span prestressed continuous rigid frame system. The specific span layout is $67.5 \mathrm{~m}+123.0 \mathrm{~m}+67.5 \mathrm{~m}=258.0 \mathrm{~m}$. The height of the bottom beam of the box girder is $7.30 \mathrm{~m}$, the height of the middle beam is $3.00 \mathrm{~m}$, and the thickness of the web is changed by three standard gradients of $0.50 \mathrm{~m}, 0.75 \mathrm{~m}$ and $1.30 \mathrm{~m}$. The thickness of the bottom plate varies from $0.30 \mathrm{~m}$ at the root to $0.854 \mathrm{~m}$ at the end according to 1.8 times parabola.

\section{Static test}

\subsection{Text content}

The static load test mainly includes: (1) The strain and stress of each bridge span control section under the test load. (2) The maximum deflection of the girder under the test load is controlled by the girder. (3) The distribution of stress and deformation of the test section under medium (partial) load and the increase coefficient of partial load.
According to the principle of equivalent internal force of the control section, 6 triaxial loading vehicles with a total weight of $400 \mathrm{kN}$ were determined through the loading trial calculation.

\subsection{The test method}

\subsubsection{Stress, strain testing}

Should be the basic principle of variable measurement, usually refers to the reservation within the scope of the standard length of $\mathrm{L}$, measured the length change of incremental average $\Delta \mathrm{L}$, by epsilon $=\Delta$ epsilon $\mathrm{L} / \mathrm{L}$ can be obtained. So the essence of the dependent variable measured is within the scope of measurement standard length $\mathrm{L}$ change incremental $\Delta \mathrm{L}$, by the transformation of the epsilon of strain and stress of sigma again can get stress value of the test section.

The transformation relation of strain and stress is:

$\sigma=\mathrm{E} \cdot \varepsilon$

In the equation:

$\sigma$ - Stress of test section under load;

E-Elastic modulus of bridge material;

$\varepsilon$ - Strain of test section under load.

\subsubsection{Deflection test}

The displacement measurement of bridge beam under test load is an important part of the bridge load test. The displacement value of each part of the bridge during the load test truly reflects the stiffness of the structure. The methods to measure the bridge displacement include digital displacement sensor measurement, level measurement and total station measurement. According to the actual situation on site, the precision level is adopted

*Corresponding author's e-mail: 429459859@qq.com 
to test the deflection of each section.

The test measuring points are arranged on the left and right sides of the sidewalk along the longitudinal deflection of the bridge. The side span is arranged as 4 measuring points, and the middle span is divided into 4 equal parts and 3 measuring points.

\subsection{Results analysis}

\subsubsection{Results of stress and strain tests}

Under the static load, the measured value $f_{e}$, the residual value $f_{p}$, the theoretical value $f_{s}$, the check coefficient $\eta$ and the relative residual deformation $f_{p}^{\prime}$ of the measured point $3 \sim 5$ at the bottom of the JM3 section beam are measured, as show in Table 1 and Figure1 2.
Table 1. Comparative Analysis of static load strain results of section (unit: $\mathrm{mm}$ )

\begin{tabular}{|c|c|c|c|c|c|c|}
\hline $\begin{array}{c}\text { loading } \\
\text { method }\end{array}$ & $\begin{array}{c}\text { Measure } \\
\text { point }\end{array}$ & $f_{e}$ & $f_{p}$ & $f_{s}$ & $\eta$ & $f_{p}^{\prime}$ \\
\cline { 2 - 7 } & $3 \#$ & 48 & 5 & 82 & 0.59 & 9.43 \\
\cline { 2 - 7 } $\begin{array}{c}\text { unbalance } \\
\text { loading }\end{array}$ & $4 \#$ & 47 & 8 & 82 & 0.57 & 14.55 \\
\cline { 2 - 7 } & $5 \#$ & 43 & 2 & 82 & 0.52 & 4.44 \\
\hline \multirow{2}{*}{$\begin{array}{c}\text { medium } \\
\text { loading }\end{array}$} & $3 \#$ & 47 & 2 & 82 & 0.57 & 4.08 \\
\cline { 2 - 7 } & $5 \#$ & 40 & 3 & 82 & 0.49 & 6.98 \\
\hline
\end{tabular}

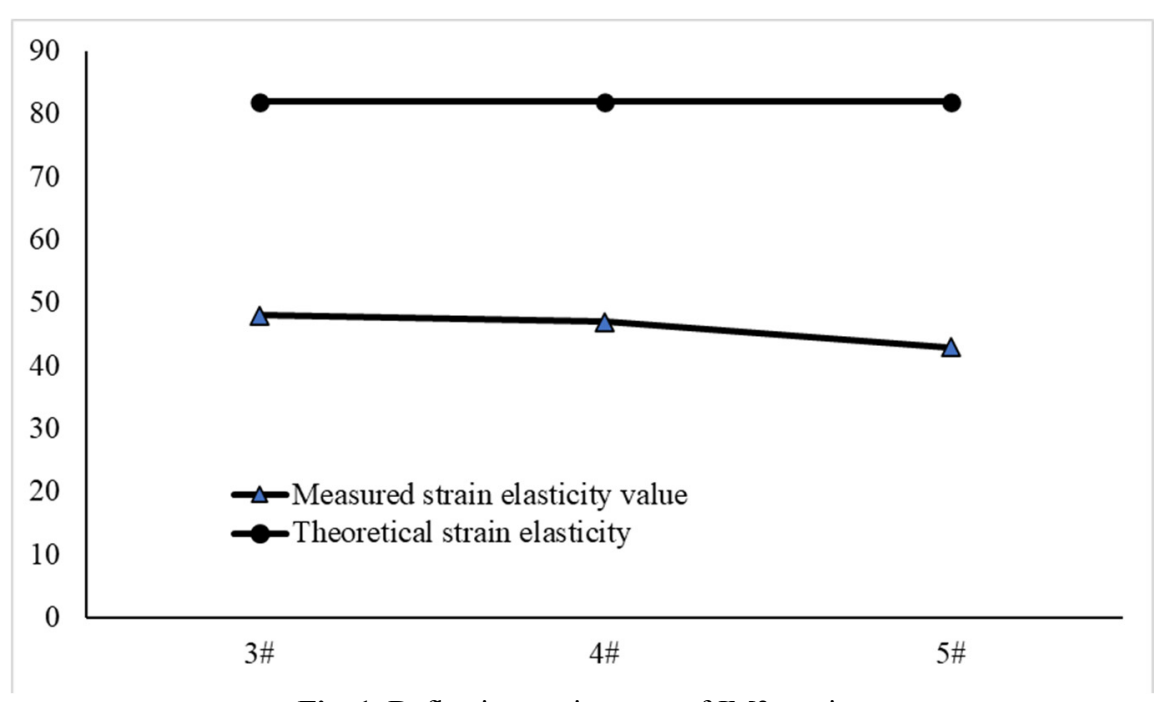

Fig. 1. Deflection strain curve of JM3 section

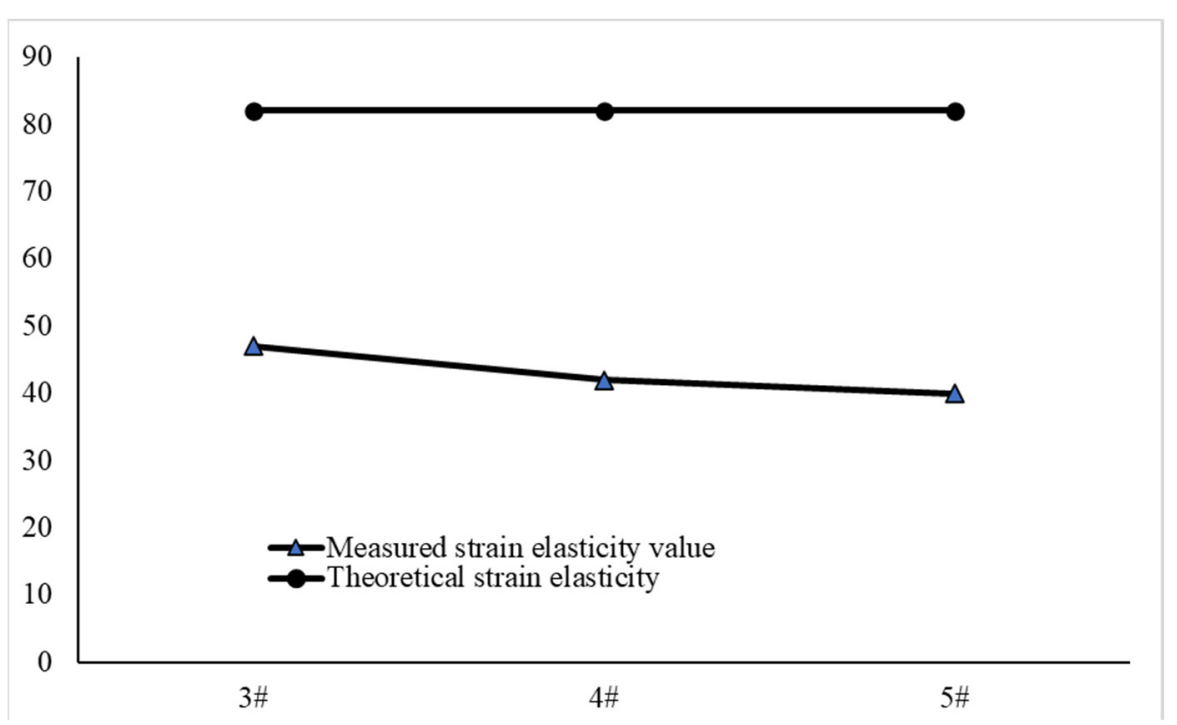

Fig. 2. Load strain curve in section JM3

As can be seen from the above chart, the bridge under test load, the overall normal JM3 test section of the measured strain, the strain calibration coefficient between
$0.49 \sim 0.59$, the overall bridge below the prescribed calibration coefficient constant between $0.6 \sim 0.9$, the actual strength is higher, the structure, the beneficial 
effects of theory or simplified calculation scheme should be safety is likely to be the cause of the calibration coefficient is smaller. After the load is removed, the measured residual strain at most measuring points is smaller than the specified limit value $(20 \%)$, indicating that the structure is in an elastic working state.

\subsubsection{Deflection test results}

The test load is JM3 cross section load, the measured points of the existing bridge are distributed along the longitudinal direction of the bridge (including upstream and downstream). The measured values $f_{e}$, residual value $f_{p}$, theoretical value $f_{s}$, check coefficient $\eta$ and relative residual deformation $f_{p}^{\prime}$ of the measured points under the action of static load are shown in Table 2 below. The deflection distribution diagram is shown in Figure 3.

Table 2. Deflection test results of Working condition 2 (JM3 medium load)

\begin{tabular}{|c|c|c|c|c|c|c|c|c|c|c|c|}
\hline \multirow{2}{*}{$\begin{array}{l}\text { loading } \\
\text { method }\end{array}$} & \multirow{2}{*}{$\begin{array}{c}\text { Distance } \\
\text { from } \\
\text { beam end } \\
(\mathrm{m})\end{array}$} & \multicolumn{5}{|c|}{ Upstream side (mm) } & \multicolumn{5}{|c|}{ The downstream side (mm) } \\
\hline & & $f_{e}$ & $f_{p}$ & $f_{s}$ & $\eta$ & $f_{p}^{\prime}$ & $f_{e}$ & $f_{p}$ & $f_{s}$ & $\eta$ & $f_{p}^{\prime}$ \\
\hline 2 & 16.88 & 1.28 & 0.11 & 2.85 & 1 & 7.91 & 1.14 & 0.16 & 2.85 & 1 & 12.31 \\
\hline 3 & 33.75 & 2.07 & 0.25 & 3.77 & 1 & 10.78 & 1.98 & 0.19 & 3.77 & 1 & 8.76 \\
\hline 4 & 50.63 & 1.45 & 0.24 & 2.53 & l & 14.20 & 1.51 & 0.15 & 2.53 & 1 & 9.04 \\
\hline 6 & 98.25 & -5.53 & -0.17 & -10.39 & 0.53 & 2.98 & -5.67 & -0.17 & -10.39 & 0.55 & 2.91 \\
\hline 7 & 129 & - & -0.26 & -23.69 & 0.46 & 2.32 & -11.21 & -0.27 & -23.69 & 0.47 & 2.35 \\
\hline 8 & 159.75 & -6.10 & -0.21 & -10.22 & 0.60 & 3.33 & -5.98 & -0.19 & -10.22 & 0.58 & 3.08 \\
\hline 10 & 207.38 & 1.57 & 0.24 & 2.48 & 1 & 13.26 & 1.52 & 0.11 & 2.48 & 1 & 6.75 \\
\hline 11 & 224.25 & 2.30 & 0.15 & 3.71 & 1 & 6.12 & 2.19 & 0.08 & 3.71 & 1 & 3.52 \\
\hline 12 & 241.13 & 1.58 & 0.17 & 2.80 & 1 & 9.71 & 1.67 & 0.07 & 2.80 & 1 & 4.02 \\
\hline
\end{tabular}

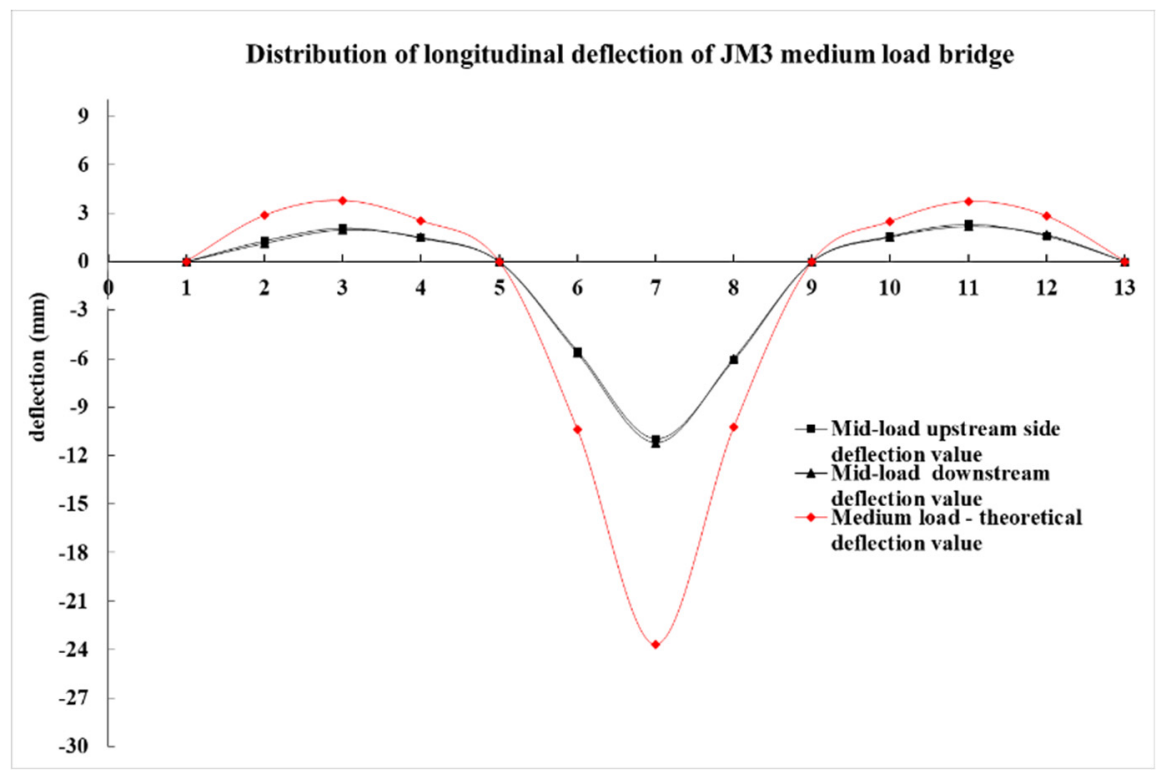

Fig. 3. Longitudinal deflection diagram along the bridge in Working condition II (JM3 medium load)

It can be seen from Table 2 3 that, under the test load of working condition $1 \sim 2$, the measured deflection value of each control section of the bridge is smaller than the theoretical value, the deflection check coefficient is between $0.42 \sim 0.62$, the maximum measured deflection increment is $10.97 \mathrm{~mm}$, which is $1 / 11212$ of the calculated span $(123 \mathrm{~m})$ and meets the requirements of the code. After unloading under various loading conditions, the measured relative residual deformation at each control point is less than the specified limit value $(20 \%)$, and the maximum value is $14.91 \%$.

\section{Conclusion}

The measured stress and deflection results show that the test coefficient of stress and deflection at each measuring 
point of the box girder does not exceed the standard limit value 1, the relative residual strain or deformation at each measuring point does not exceed the standard limit value $20 \%$, the stress condition of the tested section of the box girder is normal, and the structure is under normal stress under the action of test load.

The static load test results show that: the existing bridge load test span structure 1\#, 2\#, 3\# of the bridge box girder, under the equivalent of the design load, the stress and deflection of the test section did not appear abnormal, the structure is in the normal stress state, the overall bearing capacity of the bridge structure meet the design load (highway -I) normal use requirements.

\section{References}

1. Ministry of Transport, PRC, Highway and Bridge Carrying Capacity Test and Assessment Procedures: JTG/TJ21-2011[S].

2. Geng Bo, Zhang Xiedong, Shen Chengwu, et al. Study on static load test of long-span continuous box Girder bridge $[\mathrm{J}]$. Journal of wuhan university of technology: traffic science and engineering, 2004, 28(5):755-758.

3. Ministry of Transport, PRC, Load Test Rules for Highway and Bridge: JTG/T J21-01 -- 2015 [S].

4. Zhou An, Yang Qingyin, HAO Chengyi. Evaluation of bridge structure Performance based on static load test [J]. Engineering and construction, 2008, 22(5):695-697.

5. Guo Yuyu, Li Xiaozhen. Experimental study on static and dynamic load of the reinforced xiangqin gorge bridge [J]. Sichuan architecture,2006,26(3):107-108. 